\title{
The mutational load and a T-cell inflamed tumor phenotype identify ovarian cancer patients rendering tumor-reactive T cells from PD-1+ tumor- infiltrating lymphocytes
}

\section{Diego Salas-Benito}

Universidad de Navarra

Enrique Conde

Centro de Investigacion Medica Aplicada Ibon Tamayo-Uria

Centro de Investigacion Medica Aplicada Uxua Mancheño

Centro de Investigacion Medica Aplicada

Edurne Elizalde

Centro de Investigacion Medica Aplicada

\section{David Garcia-Ros}

Centro de Investigacion Medica Aplicada

Jose M. Aramendia

Universidad de Navarra

Juan C. Muruzabal

Complejo Hospitalario de Navarra

Julia Alcaide

Hospital Costa del Sol

Francisco Guillen-Grima

Universidad de Navarra

Jose A. Minguez

Universidad de Navarra

Jose Amores-Tirado

Hospital Costa del Sol

Antonio Gonzalez-Martin

Universidad de Navarra

\section{Pablo Sarobe}

Centro de Investigacion Medica Aplicada

Juan J. Lasarte

Centro de Investigacion Medica Aplicada 


\section{Mariano Ponz-Sarvise}

Centro de Investigacion Medica Aplicada

Carlos E. De Andrea

Universidad de Navarra

Sandra Hervas-Stubbs ( $\nabla$ mshervas@unav.es )

Centro de Investigacion Medica Aplicada https://orcid.org/0000-0003-3391-1516

\section{Research}

Keywords: Tumor-infiltrating lymphocytes, adoptive immunotherapy, ovarian cancer.

Posted Date: May 1st, 2020

DOI: https://doi.org/10.21203/rs.3.rs-25670/v1

License: (c) (1) This work is licensed under a Creative Commons Attribution 4.0 International License.

Read Full License 


\section{Abstract}

Background: Adoptive immunotherapy with tumor-infiltrating lymphocytes (TIL) may benefit from the use of selective markers, such as programmed cell death protein 1 (PD-1), for tumor-specific T-cell enrichment, as well as predictive biomarkers that help identify those patients capable of rendering tumor-reactive TIL products. We have investigated this in ovarian cancer (OC) patients as candidate for TIL therapy implementation.

Methods: PD $-1^{-}$and PD-1+ CD 8 TILs were isolated from resected ovarian tumors and, after polyclonal expansion, TIL products were tested against autologous tumor cells. Reactivity was assessed by IFNg production (ELISPOT) and upregulation of CD137. Baseline tumor samples were examined using flow cytometry, multiplexed quantitative immunofluorescence, Nanostring technology, for gene expression profile (GEP) analyses, as well as a next generation sequencing gene panel, for tumor mutational burden (TMB) calculation, to identify those features that distinguished patients with tumor-reactive and nontumor-reactive TIL products.

Results: Tumor-reactive TILs were detected in half of patients and were exclusively present in cells derived from the PD-1+ fraction. Flow-cytometric studies revealed that fresh tumors from patients rendering tumor-reactive TILs presented a significantly higher frequency of $\mathrm{CD} 137^{+}$cells within the $\mathrm{PD} 1^{+} \mathrm{CD} 8^{+}$subset. Multiplexed immunofluorescence supported this finding, which was particularly striking in intraepithelial CD8 TILs. Baseline GEP analysis showed that patients rendering tumor-reactive TILs exhibited a significantly higher T-cell inflamed signature. Despite no correlation between TMB and GEP, both parameters stratified tumors, with patients with higher TMB and/or T-cell inflamed signature score rendering tumor-reactive TILs.

Conclusion: We have demonstrated that PD-1 identifies autologous-tumor specific CD8 T cells infiltrating ovarian tumors and have uncovered predictive factors that identify $\mathrm{OC}$ patients who are likely to render tumor-reactive cells from PD-1 ${ }^{+}$TILs. These findings have important implications for improving the efficacy of TIL therapy in OC.

\section{Background}

The adoptive transfer of tumor-infiltrating T lymphocytes (TILs) has shown clinical efficacy in patients with metastatic melanoma [1] and cervical cancer [2]. TIL therapy consists of the ex vivo expansion of T cells from tumor material and transfers back into the same patient after a lymphodepleting preparative regimen. Accumulating evidence supports ovarian cancer (OC) as candidate for TIL therapy $[3,4]$. However, attempts to treat $\mathrm{OC}$ patients with TILs have been generally disappointing [5-7].

An important factor that determines the success of this therapy is the antitumor reactivity of the infused cellular product $[2,8]$. During the TIL expansion process there is an interclonal competition with different T-cell clones increasing or decreasing in frequency. Therefore, the more tumor-specific clones there are in 
the starting culture, the greater the chance of maintaining tumor-specific clones at an appreciable frequency in the final product. This may explain why the TIL therapy has yielded objective responses in cancers with high numbers of tumor-specific TILs, such as melanoma $[9,10]$ and virus-associated tumors [2], but not in cancers with lower numbers of tumor-specific TILs, such as OC [5-7].

Surface markers, such a programmed cell death protein 1 (PD-1) and CD137, have been proven to be able to identify melanoma-specific CD8 TILs $[11,12]$. The $\operatorname{CD} 8^{+} \mathrm{CD} 137^{+}$population is minority and largely confined to the CD8 ${ }^{+}$PD- $1^{+}$TIL subset. For this reason, PD-1 has been proposed as the marker that more comprehensively identifies the repertoire of tumor-specific TILs [11]. Recently, we have shown that the enrichment and separate amplification of PD- $1^{+}$CD 8 TIL improves the antitumor efficacy of TIL therapy in mouse models of solid tumors[13]. Our results were further confirmed by Jing et al. in hematological tumors[14].

Overall, these findings have important implications since PD-1 may enable the isolation of rare tumorspecific lymphocytes and allow TIL therapy to be extended to less immunogenic tumors. Several pieces of evidence suggest that PD-1 may also demarcate tumor-specific CD8 T cells in ovarian tumors. Thus, the expression of PD-1 in intraepithelial CD8 TIL has been associated with a favorable prognosis in highgrade serous carcinomas[15]. Prior studies have shown that NY-ESO-1-specific CD8 ${ }^{+}$TIL from OC patients express PD-1 [16]. In addition, ovarian tumors with deficient homologous recombination (HR) have a higher predicted neoantigen (NeoAg) load and infiltrating CD8 T cells in these tumors have increased PD-1 expression[17].

Recently, investigation of potential biomarkers that may predict sensitivity to immunotherapy has become an area of active research. In the case of TIL therapy, a number of promising biomarkers have been discovered in the infused cell product that could predict clinical response[18,19]. However, little is known about factors in the original tumor that may help identify patients able to render tumor-reactive TIL products and, therefore, that might be eligible for this therapy.

In this study, we have investigated the role of PD-1 as a selective marker for the pre-enrichment, before expansion, of tumor-specific CD8 TILs in OC. Using multiple molecular and cellular analyses, we have also examined tumors at baseline to identify parameters that help us predict from which patients we can expect to obtain tumor-specific TILs.

\section{Methods}

\section{Patients and tumor processing}

We evaluated 10 chemotherapy-naïve patients with different OC subtypes and at different cancer stages as described in Additional file 1: Table S1. After surgery, the fresh tumor underwent sterile dissection in the Anatomic Pathology Facility (Clinica Universidad de Navarra). A representative tumor sample was sent for formal pathological analysis, while geographically discrete $(0.7-1 \mathrm{~cm} 3)$ tumor fragments $(\mathrm{n}=2-3)$ were used for tumor cell and TIL isolation after mechanical and enzymatic digestion (Additional file 1: 
Fig. 1). Fresh tumor fragments were minced under sterile conditions and dissociated into single-cell suspensions using the human Tumor Dissociation Kit and the gentleMAC dissociator (Miltenyi, Bergisch Gladbach, Germany). An aliquot of the cell suspension was analyzed by flow cytometry (FC) and the remaining cells were magnetically labeled with non-tumor cell depletion cocktail A from the human Tumor Cell Isolation Kit (Miltenyi). Negative and positive fractions were sorted using an LS Columns (Miltenyi) and separately cryopreserved until further analysis. The positive fraction containing non-tumor cells was used for TIL isolation. Negative fraction enriched in tumor cells was used as autologous tumor targets for TIL reactivity testing.

\section{Flow-cytometric analysis}

Tumor single-cell suspensions were incubated with Zombie NIR (ZN) Fixable dye (Biolegend, San Diego, $\mathrm{CA}$, USA) and were, subsequently, stained with the following fluorochrome conjugated monoclonal antibodies (mAbs), as indicated: EPCAM-FITC (9C4), CD45-PECy7 (HI30), CD3-PercPCy55 (SK7), CD4BV421 (RPA-T4), CD8-BV510 (SK1), PD-1-PE (EH12.2H7) and CD137-APC (4B4-1) (Biolegend) in FACS buffer [phosphate buffered saline (PBS) without $\mathrm{Ca}^{2+} / \mathrm{Mg}^{2+}$, heat-inactivated fetal calf serum $(0.5 \%$, SIGMA, San Luis, Misuri, USA), EDTA (2 mM, GIBCO, Dublin, Ireland) containing Beriglobin P $(10 \mu \mathrm{g} / \mathrm{ml}$, CSL Behring GmbH, Marburg, Germany) to block Fc receptors. Cells were acquired in a FACSCanto-II cytometer (BD Biosciences, Franklin Lakes, NJ, USA) and analyzed using FlowJo software (BD Biosciences).

\section{TIL isolation and expansion}

Non-tumor cell-enriched fractions were thawed and rested overnight in T-cell media [1:1 mix of RPMI1640-glutamax (Gibco) and AIMV (Gibco), supplemented with 5\% heat-inactivated human serum (SIGMA), $12.5 \mathrm{mM} \mathrm{HEPES,} 100 \mathrm{U} / \mathrm{ml}$ penicillin, $100 \mu \mathrm{g} / \mathrm{ml}$ streptomycin, and $10 \mu \mathrm{g} / \mathrm{ml}$ gentamicin (Gibco)]. The next day, cells were stained with fluorochrome conjugated mAbs against CD8 (RPAT-8 or SK1) and PD-1 [EH12.2H7], and 7-amino-actinomycin D (dead cell marker) (Biolegend) and were sorted into PD-1 negative (PD-1 $)$ and PD-1 high (PD-1 ${ }^{\text {hi }}$ ) CD8 ${ }^{+}$T cells using a FACSAria cell sorter (BD Biosciences). Isolated cells were separately expanded using the "rapid expansion protocol" (REP) in T-cell media containing soluble anti-CD3 mAb (OKT3) $(30 \mathrm{ng} / \mathrm{ml}$, Biolegend), human IL-2 (3,000 IU $/ \mathrm{ml}$, Proleukin, Prometheus Laboratories Inc., San Diego, CA, USA), and $3 \times 10^{7}$ irradiated peripheral blood leucocytes pooled from 3 different donors. After 12-15 days of expansion, $T$ cells were cryopreserved until further analysis.

\section{TIL reactivity assessment}

Expanded CD8 TILs and autologous tumor-enriched cells were thawed and separately rested overnight in T-cell media without IL-2. The next day, TILs $\left(5^{\prime} 10^{4}\right.$ cells/well) were co-cultured either alone or with target cells [autologous tumor-enriched cells or the $\mathrm{H} 929$ plasmacytoma cell line, as unrelated tumor cells] (10 5 cells/well) in ELIIP plates (Millipore, Burlington, Massachusetts, USA) coated with purified anti-human 
interferon(IFN)-g mAb (Mabtech, Stockholm, Sweden), in the presence or absence of Human leukocyte antigen (HLA)-I blocking mAb (W6/32, Bio-x-cell, West Lebanon, NH, USA). Thirty-six hours later, cells were lysed with water, washed with PBS-Tween-20 $(0.1 \%)$ and the plate was developed using biotinilated antiIFN-g mAb (7-B6-1) (1h, RT), streptavidin-ALP (1h, RT) and BCIP/NBT substrate (20', RT) (Mabtech). Results were analyzed using a CTL-ImmunoSpot S6 MicroAnalyzer (Cellular Technology, Cleveland, $\mathrm{OH}$, USA). Data are expressed as the number of spot-forming cells (SFC) per $5^{\prime} 10^{4}$ For each patient, the number of target-specific IFN- $\gamma$ SFC was determined by calculating the difference between the number of spots generated in the absence (TILs alone) or presence of target cells (autologous tumor or unrelated tumor). Reactivities $>30$ specific IFN- $y$ SFC and twice the background were considered positive. When autologous tumor-enriched cells were available, the reactivity of TILs was confirmed by assessment of CD137 upregulation. Briefly, TILs $\left(1 \times 10^{5}\right.$ cells/well) were cultured, either alone or with target cells (0.5$1 \times 10^{5}$ cells/well), in T-cell media. Twenty-four hours later, cells were incubated with Zombie NIR Fixable dye. Then, they were stained with anti-human CD45-PECy7 (HI30), CD3-PercPCy55 (SK7), CD8-APC (SK1) and CD137-PE (4B4-1) mAbs (Biolegend), in the presence of Beriglobin P, and analyzed by FC. CD8 TILs were identified as blastic $\mathrm{CD} 45^{+} \mathrm{CD} 3^{+} \mathrm{CD} 8^{+}$living cells. $\mathrm{H} 929$ cells were acquired in 2017 from ATCC (CRL9068) and used to produce the working bank that was cryopreserved in N2L. Cells with 1-4 passages from the working bank were used in the experiments. H929 cells were certified as being Mycoplasma-free by using the MycoAlert Mycoplasma Detection Kit (Lonza, Basel, Switzerland).

\section{Multiplex immunofluorescence staining and analysis}

Multiplex immunofluorescence (IF) staining, validation and analysis are detailed in additional file 1: Supplementary Methods and Fig. S2 and S3. Briefly, 4- $\mu \mathrm{m}$ sections of formalin-fixed paraffin-embedded (FFPE) tissue samples were deparaffinized and antigen retrieval was performed using DAKO PT-Link heat induced antigen retrieval with a low $\mathrm{pH}(\mathrm{pH} 6)$ or high $\mathrm{pH}(\mathrm{pH}$ 9) solution (Agilent, Santa Clara, $\mathrm{CA}, \mathrm{USA}$ ). Samples were stained with mAbs targeting CD4 (4B12, Agilent), CD8 (C8/144B, Agilent), FOXP3 (236A/E7, Abcam, Cambridge, UK), PD1 (NAT105, Cell Marque, Rocklin, CA, USA), CD137 (BBK-2, ThermoFisher Scientific Waltham, MA, USA), cytokeratin (CK) (AE1/AE3, Leica Biosystems, Wetzlar, Germany). Visualization was performed with the Opal 7-Color Automation IHC Kit (Akoya Biosciences, Menlo Park, CA USA). Imagery was acquired on a Vectra-Polaris Automated Quantitative Pathology Imaging System (Akoya Biosciences) and analyzed using InForm 2.4.8 (Akoya Biosciences).

\section{Somatic mutation estimation}

The mutational load was estimated using the Trusight Tumor 170 panel (TST170) from Illumina (San Diego, CA, USA). DNA was isolated from FFPE sections. One cut section from each tissue sample was stained with Hematoxylin and Eosin (H\&E) and assessed for sample quality. Each H\&E-stained slide was reviewed by a pathologist to evaluate the adequacy of tumor representation, the quality of tissue preservation, and whether significant artifacts relating to fixation, processing, or prefixation tissue handling were present. In samples from patients 3 and 10, laser capture microdissection was performed to enrich specimens with tumor tissue, under direct microscopic visualization. 10 to 205 - $\mu$ m-thick 
sections were used for DNA isolation. DNA extraction, library preparation and sequencing (NextSeq 500 using high-output cartridge and v2 chemistry) were performed by Macrogen according to Illumina's instructions. Sequence alignment and variant calling were performed using the TST170 BaseSpace application. Variant annotation was performed using Annovar (dbSNP150, 1000G and COSMIC). To assess the number of somatic mutations only missense_variants and

missense_variant\&splice_region_variants were considered. Germline filtering was performed by filtering Variant Allele Frequency (VAF) between 0.4-0.5 and $>0.9$. Polymorphisms were excluded by filtering against $1000 \mathrm{G}$ using a cutoff of minor allele frequencies of $<0.0002$. Tumor mutational burden (TMB) was calculated as the number of somatic mutations per 0.524 Megabase pairs (the targeted genomic regions of TST170). Multiple genomic alterations in different patients were visualized using the oncoPrint function (R package ComplexHeatmap).

\section{NanoString-based gene expression profiling}

After histopathological evaluation to certify the tumor content, five 5 - $\mu$ m-thick unstained FFPE sections were obtained from each tumor block. Samples were deparaffinated with xylene and digested with proteinase K and total RNA was extracted with the RNeasy FFPE kit (QIAGEN, Hilden, Germany) following the manufacturer's protocol. nCounter PanCancer Immune Profiling panel analysis (Nanostring, Seattle, Washington, USA) was performed by IMIBIC (Cordoba, Spain) following the manufacturer's instructions. Analyses of expression were performed using nSolver Analysis Software (v 4.0), with normalization utilizing positive and negative control probes, as well as the most stable housekeeping (HK) genes across samples. $p$-values $<0.05$ were considered to identify differentially expressed genes (DEG). Volcano-plots and unbiased clustering of DEG were generated using R (v3.5.3). For analysis of immune signatures, after HK normalization, a log10 transformation was applied, and the signature score was calculated by averaging the expression level of those genes included in (i) IFN-y signature (6-gene: CXCL10, CXCL9, HLA-DRA, ID01, IFNG, STAT1), (ii) a modified Expanded immune signature [including 15 genes (CCL5, CD2, CD3D, CD3E, CXCL10, CXCL13, CXCR6, GZMB, GZMK, HLA-DRA, HLA-E, ID01, IL2RG, LAG3, STAT1) of the original 18 genes, and (iii) a modified T-cell inflamed signature [including 16 genes (CCL5, CD27, CD274, CD276, CD8A, CMKLR1, CXCL9, CXCR6, HLA-DQA1, HLA-E, ID01, LAG3, PDCD1LG2, PSMB10, STAT1 and TIGIT) of the original 18 genes[20]. Three (CIITA, NKG7, TAGAP) and two (NKG7 and HLADRB1) genes were not included in the Expanded immune signature and the T-cell inflamed signature, respectively, because they were absent in the PanCancer Immune Profiling Panel.

\section{Statistical analysis}

GraphPad Prism 6 software was used for graphic representation and most statistical analysis. Data from multiplexing IF were analyzed using STATXact (Cytel Studio version 11.0.0). We used the Friedman test to identify differences among the 10 images of the same patient. If no statistically significant differences were found, then the images were considered homogeneous and the average frequency of each cell subset studied was calculated. To compare between reactive and non-reactive group we used nonparametric Mann-Whitney test, two-tailed (FC, TIL reactivity, mutational load, gene signature score) or 
one-tailed (multiplexing IF) with 95\% confidence interval. Detailed information is included in each figure legend.

\section{Results}

\section{TILs in fresh ovarian tumors display variable expression of PD-1 and CD137}

Single-cell suspensions of fresh human ovarian tumors comprised both $\mathrm{CD} 45^{+}$cells and $\mathrm{EpCAM}^{+}$cancer cells (Fig. 1 and Additional file 1: Fig. S4). The percentages of $\mathrm{CD} 4^{+}$and $\mathrm{CD} 8^{+}$cells within the $\mathrm{CD} 45^{+}$ population (Additional file 1: Fig. S5A) ranged from 3.6 to $36.1 \%$ and from 5.9 to $31.6 \%$, respectively, with no differences among them. CD ${ }^{+}$and $\mathrm{CD} 8^{+}$TILs expressed PD-1 at variable levels (range of $1.73-72.7 \%$ and of $0.1-88.6 \%$ for $C D 4^{+}$and $C D 8^{+}$cells, respectively) (Fig. 1 and Additional file 1: Fig. S5B). No differences were found in the percentage of $\mathrm{PD} 1^{+}$cells within $\mathrm{CD} 4^{+}$and $\mathrm{CD} 8^{+} \mathrm{TILs}$. Expression of CD137 was much lower than that of PD-1 and confined to the PD-1 ${ }^{+}$subsets. Interestingly, CD137 in CD ${ }^{+}$TILs were almost exclusively expressed on PD- ${ }^{\text {hi }}$ cells. There were no differences in the percentage of CD137 between $\mathrm{PD} 1^{+} \mathrm{CD} 4^{+}$and $\mathrm{PD} 1^{+} \mathrm{CD} 8^{+}$TILs (Additional file 1: Fig. S5C).

\section{CD8 TIL reactivity against autologous tumor was confined to the PD- $1^{\text {high }}$ compartment}

To determine if PD-1 may enrich tumor-specific T cells in OC, we isolated CD8 TILs with extreme expression of PD-1, namely PD-1 ${ }^{-}$and PD- $1^{\text {hi }}$ cells, from 10 resected ovarian tumors and expanded them separately. The number of isolated PD- $1^{-}$and PD- $1^{\text {hi }}$ CD 8 TILs varied among patients. Both subsets expanded efficiently (Additional file 1: Table S2). Next, we tested the ability of the expanded cells (also referred to as TIL products) to recognize the autologous tumor using the enriched tumor-cell fraction obtained from enzyme-digested tumors. TIL products were cultured alone or together with autologous tumor cells or unrelated tumor cells (H929) in the presence or absence of HLA-I blockade. Figure 2 shows data from patient P05. Notably, cells derived from the PD- ${ }^{\text {hi }}$ CD8 TIL subset, but not from the negative counterparts, were TR cells, as determined by IFN-y secretion (Fig. 2A) and CD137 upregulation (Fig. 2B). We found TR TILs in 5 out of 10 patients (Fig. 2C). Remarkably, the antitumor reactivity was harbored by cells derived from the PD- ${ }^{\text {hi }}$ compartment, as deduced by IFN-y ELISPOT. Recognition of autologous tumor by PD-1 ${ }^{\text {hi }}$-derived cells was HLA-I-restricted (Fig. 2 and 3). Only PD-1 ${ }^{-}$derived cells from patient P06 were able to recognize autologous tumor but this recognition was not HLA-I-restricted. Recognition was tumor specific since TILs did not respond to unrelated H929 tumor cells (Fig. 2 and 3). Our data indicate that, although only PD-1 ${ }^{+}$-derived T cells were able to recognize autologous tumor cells, the ability of PD1-selected cells to render TR TIL products varied among patients. Accordingly, patients were divided into two groups: patients with tumor-reactive (TR) TILs (P01, P02, P04, P05 and P06) and patients with nontumor-reactive (NTR) TILs (P03, P07, P08, P09, P10).

Detection of $\mathrm{CD} 137^{+}$cells within the PD- ${ }^{\text {hi }} \mathrm{CD} 8^{+}$TIL subset in the fresh tumor correlates with the antitumor reactivity of the final TIL product 
We retrospectively analyzed FC data from tumor infiltrates to find any feature that distinguished patients from the TR and NTR groups. No statistically significant differences were found in the percentage of CD $45^{+}$cells between both groups (Fig. 3A). Notably, those patients delivering TR TILs presented a significantly higher frequency of $\mathrm{CD} 8^{+}$and $\mathrm{CD} 4^{+}$lymphocytes in the fresh tumor (Fig. 3A and B). These patients also showed a higher proportion of PD $-1^{+} \mathrm{CD} 8^{+}$and PD $-1^{+} \mathrm{CD} 4^{+}$TILs (Fig. 3A). Interestingly, while no difference was found in the percentage of $C D 137^{+} P D-1^{+} C D 4^{+}$cells, those patients who rendered $T R$ TILs showed a higher proportion of CD $137^{+} \mathrm{PD}-1^{+} \mathrm{CD} 8^{+}$TILs (Fig. 3A). These patients also presented a higher frequency of $\mathrm{CD} 137^{+}$cells within the $\mathrm{PD}-1^{\mathrm{hi}} \mathrm{CD} 8^{+}$population, the cell subset sorted for further expansion (Fig. 3B). No differences were found either in the percentage of PD $-1^{+}$cells within the CD8 ${ }^{+}$ and $\mathrm{CD} 4^{+} \mathrm{TIL}$ compartment, or in the percentage of $\mathrm{CD} 137^{+}$cells within the $\mathrm{PD}-1^{\text {hi }} \mathrm{CD} 4^{+}$population (Fig. 3B).

\section{Patients with TR TIL products exhibited a higher percentage of $\mathrm{CD} 137^{+} \mathrm{PD}-1^{+} \mathrm{CD} 8^{+}$TILs in the tumor epithelium.}

We subsequently analyzed tumors by multiplexed IF (4). Representative fluorescence images from patients P05 (TR group) and P07 (NTR group) in figure 4A show the presence of CD $4^{+}$and CD8 ${ }^{+} \mathrm{T}$ cells in the tumor infiltrate. $\mathrm{CD} 4^{+}$and $\mathrm{CD} 8^{+}$TILs were more prominent in the tumor stroma than in the tumor epithelium (Fig. 4B and C). PD-1, CD137 and forkhead box P3 (Foxp3) expression was also detected in the tumor infiltrate, with PD-1 being the most notable marker (Fig. 4A).

Since FC analyses showed that patients who rendered TR TILs had a higher frequency of CD $137^{+}$PD$1^{+} \mathrm{CD} 8^{+}$cells in the tumor, we also analyzed this phenotype by multiplexed quantitative IF. Total CD $4^{+}$and CD8 ${ }^{+}$TILs, PD $-1^{+} \mathrm{CD} 4^{+}$and PD $-1^{+} \mathrm{CD} 8^{+}$TILs (regardless of whether or not they expressed CD137) and CD $137^{+} \mathrm{PD}-1^{+} \mathrm{CD} 4^{+}$and $\mathrm{CD} 137^{+} \mathrm{PD}-1^{+} \mathrm{CD} 8^{+} \mathrm{TIL}$ subsets were quantified (Fig. 4B and C). Patients with TR TILs products exhibited a slightly higher, but statistically significant, percentage of CD $137^{+} \mathrm{PD}-1^{+} \mathrm{CD} 8^{+}$ TILs in the tumor epithelium (Fig. 4C).

\section{Antitumor reactivity of final TIL products was associated with higher mutational load}

In order to determine if the TMB was related to the antitumor reactivity of the final TIL products, we estimated the mutational load of our patients using the TST170 panel. Of the 2068 variants identified (Additional file 2: Table S3), only 63 met the criteria for being somatic mutations (Additional file 2: Table S4), with a median of 4.5 mutations per patient and a range between 2 and 13 (Additional file 2: Table S5). Mutations were distributed along 39 genes (Fig. 5A). TP53 was the most commonly mutated gene, consistent with the genome landscape of OC[21]. The median of the estimated TMB was 8.6, ranging from 3.8 to 24.8 (Additional file 2: Table S5). Remarkably, those patients with the highest TMB values (TMB >20) (P02, P05 and P06) belonged to the group with TR TILs (Additional file 2: Table S5). The TMB values of the TR group were slightly but statistically significantly higher than those of the NTR group (Fig. $5 B)$. 
We also analyzed mutations in genes from the DNA damage repair (DDR) system (Additional file 2: Table S7). Twenty-eight genes out of about 120 genes involved in direct DDR[22] were contained in the TST170 panel (Additional file 2: Table S8 and Appendixes 1 and 2). Eighteen out of 28 genes appeared mutated in our patients (Additional file 1: Fig. S6A and Additional file 2: Table S8). A total of 53 variants were detected (5.3 \pm 1.9 ) affecting 8 different DDR pathways (Additional file 2: Table S9). Patients differed in the number and type of variants (Additional file 1: Fig. S6A), as well as in the number and type of pathways affected (Additional file 1: Fig. S6B). Notably, those patients with the highest TMB values (P02, P05 and P06) presented the greatest number of variants in DDR genes (Additional file 1: Fig. S6B). Patients P02 and P06 also stood out for exhibiting the greatest number of altered pathways. In addition, patients P02 and P05 showed mutations in genes involved in the Mismatch excision repair (MMR) process (Additional file 1: Fig. S6B). Strikingly, the TMB values were directly related to the number of alterations in the DDR system (Additional file 1: Fig. S6C).

In summary, alterations in the DDR system may explain the different mutational load observed in our patients. Whereas high values of TMB seemed to be related to the antitumor reactivity of the TIL products, the fact that patients with relatively low TMB values also gave TR TILs suggest that other mechanisms independent of neoantigenicity might also account for the capability of rendering TR TILs.

\section{Immune activation gene signature at the tumor site identified patients rendering TR TILs}

Gene signatures of activated T cells have been shown to predict response to anti-PD-1 therapy[20]. In order to determine if the gene expression profile (GEP) may also predict patients capable of eliciting TR TIL products, we analyzed the expression of 770 immune-related genes in 8/10 patients with FFPE-tumor RNA available. Four ovaries with non-malignant disease were included as a control. Transcriptome analysis revealed a set of 36 DEG between the TR and NTR groups (Fig. 6A). Unsupervised hierarchical clustering clearly separated patients from TR and NTR group, as well as the control ovaries (Fig. 6B).

Gene Ontology analysis identified biological processes and pathways relevant to the antitumor response that were significantly overrepresented in tumors from the TR group (Additional file 1: Fig. S7A and B). Interestingly, high baseline expression of genes involved in antigen processing/presentation [PSMB9, TAP1, TAP2, HLA-I (A, B and C) and NLRC5], T-cell activation [CD247 (CD3z), CD3E, CD3D, LCK, CD7, CD38 and ICAM3], proliferation/differentiation of cytotoxic T cells (IL2RB and IL2RG), cytotoxic activity (PRF1 and GZMB), IFN-y signaling pathway (STAT1), chemo-attraction (CXCL9, CXCL13, CXCR3 and CCR5), as well as inhibitory mechanisms (HLA-G, FOXP3 and TIGIT), was associated with TR TIL products (Fig. 6B). In addition, patients with TR TIL products exhibited a higher expression of the TNFRSF9 (CD137) transcript that was close to statistical significance (Additional file 1: Fig. S7C). In contrast, higher expression of genes involved in wound healing (COL3A1, FN1, THBD, THBS1 and TNFRSF1A), mesenchymal transition and cell adhesion (COL3A1 and FN1), angiogenesis and blood vessel development (COL3A1 and THBS1), invasiveness (DOCK9 and THBS1), as well as NF-Kb [IKBKB and TNFRSF1A (TNFR1)] and Ras-MAPK (LRRN3) pathways, was associated with NTR TIL products. 
Recently three immune-related gene expression signatures that predict clinical response to PD- 1 blockade across multiple cancer types have been identified: the IFN-g signature, the Expanded immune gene signature and the T-cell inflamed signature[20]. Interestingly, patients rendering TR TIL products exhibited significantly higher scores for the three mentioned signatures, in particular for the T-cell inflamed GEP (Fig. 6C).

Although the correlation between TMB and GEP was low, both parameters stratified TR and NTR patients (Fig. 6D). Therefore, patients with high values of either TMB (P02, P05 and P06) or GEP (P04) rendered TR TIL products, whereas those with low values of both biomarkers (P03, P07, P08 and P10) did not.

Finally, we also analyzed the expression of Cancer testis antigens (CTA) included in the panel. No differentially expressed CTA genes were detected between the TR and the NTR groups (Additional file 1: Fig. S7C). Curiously, patient P05, who rendered highly TR TILs (Fig. 2), stood out for expressing a large number of CTA genes.

\section{Discussion}

Adoptive immunotherapy using TILs in patients with melanoma [1] and cervical [2] cancer frequently results in durable complete responses. However, in OC patients, responses to TIL therapy have been less dramatic [5-7] due in part to technical difficulties in identifying and expanding the TR TIL subset. In an effort to improve TIL therapy in OC, in this study we have investigated the ability of PD-1 marker for ovarian tumor-specific T-cell enrichment. We have found that autologous tumor-specific T cells were exclusively present in cells derived from PD $-1^{+} \mathrm{CD} 8^{+}$TILs, but not from their PD1- counterparts. These findings provide a method to pre-select ovarian tumor-specific $T$ cells directly from tumor infiltrates, without the need for complex immunological screening technologies that require antigen prediction/identification to evaluate T cells. Recently, it has been shown that PD-1 also allows the identification of tumor-specific T cells in MMR-proficient gastrointestinal cancer patients [23]. This finding together with our results indicate that the utility of PD-1 to select for tumor-specific T cells is not restricted to highly immune-reactive tumors $[11,13,14,24]$.

However, only in 5 out of 10 patients studied the PD-1+-derived cells were able to recognize autologous tumor. Interestingly, those patients with TR TIL products exhibited a higher frequency of $\mathrm{CD} 137^{+}$cells within the sorted PD $-1^{+} \mathrm{CD} 8^{+}$population. Our findings reopen the debate on which marker, PD-1 or CD137, best identifies the repertoire of tumor-specific $T$ lymphocytes $[11,12]$. In the only patient studied, Ye et al. found that PD- $1^{+} \mathrm{CD} 137^{+}$cells, but not the PD $-1^{+} \mathrm{CD} 137^{-}$subset, harbored tumor-specific $\mathrm{T}$ cells in OC [12]. These results appear to contradict Rosenberg group's findings in melanoma [11], which showed that both population comprised tumor-specific CD8 TILs. CD137 expression is an indication of ongoing or recent activation of T cells [25], in contrast to PD-1 expression, which is maintained long after antigen recognition. On the other hand, the PD-1 ${ }^{+}$TIL population is heterogeneous and houses bystander and tumor-specific $T$ cells [26], some of which may be recently activated cells $\left(P D-1^{+} C D 137^{+}\right)$, whereas the large majority are cells activated long ago (PD-1 $\left.{ }^{+} \mathrm{CD} 137^{-}\right)$. The ratio between bystander and tumor- 
specific TILs vary between tumors [27], and this could explain why in OC, with smaller numbers of tumorspecific lymphocytes in comparison to melanoma [11], only $\mathrm{CD} 137^{+}$cells [12] or PD-1 ${ }^{+}$cells containing high proportion of $\mathrm{CD} 137^{+}$cells (our study) were able to render TR TILs. A more exhausted state of PD-1 ${ }^{+}$ tumor-specific TILs in OC may also account for the difficulty of obtaining TR TIL products from this population. Patient-related factors may also be implicated. Thus, similar to what we have found in OC, the ability of PD-1 to enrich for tumor-specific TILs in melanoma patients has also been shown to be inconsistent[24].

Therefore, the use of PD-1 as a single-marker to isolate tumor-specific cells appears to be controversial. On the other hand, the expression of CD137 is transitory. Consequently, a large proportion of tumorspecific T cells may be missed in the CD137- fraction. A recent study in colorectal and lung cancer has shown that CD39 accurately distinguishes between tumor-specific (PD- $\left.1^{+} \mathrm{CD} 39^{+}\right)$and bystander (PD$1^{+}$CD39-) TILs[26]. In another study, co-expression of PD-1 with markers of tissue-resident memory cells, such as CD39 and CD103, seemed to identify tumor-specific TILs[28]. Presumably, combinations of PD-1 with other markers would be necessary to accurately identify the full repertoire of tumor-specific TILs, especially in tumors in which these cells are rare.

The finding that those patients rendering TR TILs had a higher frequency of CD $137^{+} \mathrm{PD}-1^{+} \mathrm{CD} 8^{+} \mathrm{TILS}$ located in the tumor epithelium, suggests that their tumors may have special features that have enabled the activation of $T$ cells, e.g. the presence of relevant antigens or/and an immune-permissive tumor microenvironment (TME). TMB provides an indirect assessment of tumor antigenicity because a high level of mutations will increase the likelihood of neoAg generation. The TMB values in our patients, as estimated using the TST170 panel, ranged from 3.8 to 24.8 mutations/Mb. These values were within the range of those found for OC using whole-exome sequencing[29] and FoundationOne[30], although the median values ( 8.6 mutations/Mb) were higher than those estimated with these methods (3-4 mutations/Mb)[29,30]. The use of a small gene panel and the low number of patients studied may have overestimated the median TMB values[31]. Remarkably, those patients with the highest TMB levels (P02, P05 and P06) belonged to the group with TR TILs. These patients also presented co-mutations in genes from the DDR system, which may explain their higher TMB. Interestingly, P02 and P05 showed mutations in genes from the MMR pathway that has been associated with high TMB values across several cancer types[32]. Mutations in the DDR system predict clinical benefit from ICls treatment[33-36]. In addition, comutations in different DDR pathways have also been associated with increased TMB and activated T-cell infiltration in OC[37].

The finding that some patients from the TR group showed relatively low TMB values and DDR mutations suggests that mechanisms independent of neoantigenicity might also account for the capability of rendering TR TILs. The expression profile of immune-related genes reflects the nature of the TME, which is also an important feature of cancer immunobiology. Interestingly, tumors from patients rendering TR TILs showed a high expression of genes involved in antigen processing/presentation, T-cell activation, proliferation/differentiation of T cells, cytotoxic activity, IFNY-signaling, chemo-attraction, as well as 
mechanisms to regulate T-cell response. This GEP is characteristic of immune-reactive tumors with prolonged survival and response to ICls[33,38]. A similar GEP has also been associated with high tumor Tcell infiltration in OC patients[39,40]. In line with the transcriptomic data, FC analysis of tumor infiltrates revealed a significantly higher frequency of CD8 and CD4 T cells in those patients who rendered TR TILs. Likewise, a high expression of genes involved in wound healing, mesenchymal transition, extracellular matrix remodeling, angiogenesis and invasiveness, was associated with the lack of antitumor reactivity of the final TIL product. The concurrent over-expression of genes involved in these processes has been associated with the presence of immunosuppressive TME[41], T-cell dysfunctionality[42] and resistance to anti-PD-1 therapy[33].

Three multigene expression signatures (IFN- $y$, Expanded-immune and T-cell-inflamed signature) have been associated with clinical benefit from anti-PD-1 treatment in several cancer types[20]. Importantly, patients rendering TR TIL products exhibited significantly higher scores for the three mentioned signatures, in particular for the T-cell inflamed GEP. These findings suggest that GEP may represent a useful tool to identify patients who are likely to render TR TILs and, ultimately, who may benefit from TIL therapy.

Interestingly, while the TMB values did not correlate with the GEP score, both parameters stratified TR and NTR groups. Thereby, patients with high values of either TMB and/or GEP rendered TR TIL products, whereas those with low values of both TMB and GEP did not. Our data show some similarities with the predictive value of TMB and GEP to anti-PD1 therapy across different tumor types[43,44]. The ability of these two parameters to reflect overlapping but not always coincident aspects of tumor immunology may explain their lack of correlation.

The fact that patients with relatively low TMB rendered TR TILs suggests that the quality of neoAgs, but not the quantity[45], or the presence of relevant shared tumor-associated antigens may have determined the antitumor reactivity of the final TIL product. In the case of OC, several groups have identified TILS specific for private[46] and hot-spot mutations[47], as well as for CTA[4,16]. Interestingly, the patient who in our study stood out for rendering highly TR TILs (P05), exhibited a high expression of very potent CTA, such as NY-ESO-1, MAGEC2 and TPTE. In the future, it will be interesting to determine what type of antigens account for the antitumor reactivity of TIL products in OC.

\section{Conclusions}

Although our findings will need to be validated using a larger cohort of patients, they provide a rationale for further exploring the utility of biomarkers to (i) identify and selectively expand tumor-specific TILs, and (ii) serve as guides for selecting patients who are likely to render TR TILs. In order to increase the tumor reactivity of the final TIL product, other selective markers may be considered together with PD-1 to distinguish tumor-specific from bystander T cells. The combination of several parameters, such as TIL density and the presence of intraepithelial $\mathrm{CD} 137^{+}$cells in the fresh tumor together with the GEP and the $T M B$, could guide future steps to defining an extensive predictive biomarker panel to identify patients 
eligible for TIL therapy. The use of such a predictive biomarker panel together with newer ways to select tumor-specific TIL, will help to extend this cell therapy to less immune-reactive tumors, such as OC.

\section{List Of Abbreviations}

CTA: Cancer testis antigens

CK: Cytokeratin

DEG: Differentially expressed genes

DDR: DNA damage repair

FC: Flow cytometry

Foxp3: Forkhead box P3

FFPE: Formalin-fixed paraffin-embedded

GEP: Gene expression profile

HR: Homologous recombination

HK: Housekeeping

HLA: Human leukocyte antigen

IFN: Interferon

MMR: Mismatch excision repair

mAbs: Monoclonal antibodies

IF: Multiplex immunofluorescence

NeoAgs: Neoantigens

NTR: Non-tumor-reactive

OC: Ovarian cancer

PD-1: Programmed cell death protein 1

REP: Rapid expansion protocol

TME: Tumor microenvironment 
TMB: Tumor mutational burden

TIL: Tumor-infiltrating lymphocytes

TR: Tumor-reactive

VAF: Variant Allele Frequency

\section{Declarations}

\section{Ethics approval and consent to participate}

The study was approved by the Ethics and Scientific Committee of the Clinica Universidad de Navarra and Hospital Costa de Sol (2016.145). Written informed consent was obtained from all patients. Samples and data from patients included in the study were provided by the Biobank of the University of Navarra and Hospital Costa del Sol and were processed following standard operating procedures approved by the Ethics and Scientific Committees.

\section{Consent for publication}

Not applicable.

\section{Availability of data and materials}

All data generated or analyzed during this study are included in this published article and its supplementary files.

\section{Competing interests}

The authors declare no potential conflicts of interest

\section{Funding}

SHS is founded by ISCIII/FEDER, UE (PI15/02027, PI18/00556); MCIU-AEI/FEDER, UE (RTC-2017-6745-1); AECC-Semilla (4189), Gobierno de Navarra [Ayudas a Centros Tecnológicos (PT039 EstraTeC) and Departamento de Salud (045-2017)]. EC, JJL and SHS are funded by Fundación Ramón Areces. CEA and DGR are funded by the Instituto de Salud Carlos III (AC14/00034) y co-financed by Fondos FEDER (Spain).

\section{Authors' contributions}

DSB and MPS were involved in study conceptualization, acquired ethical approvals for the study and curated patient data. DBS also analyzed FC, multiplex IF and genetic data, and wrote the original manuscript draft. EC and EE performed DNA and RNA isolation from FFPE tissues. EC and ITU performed bioinformatics and statistical analysis and analyzed publicly available datasets. UM and EE performed 
tissue processing, cell isolation and expansion, FC staining and TIL reactivity assays. DGR performed multiplex IF staining. JMA, JA, JAM, JAT were involved in tissue procurement. FGG was involved in statistical analysis. JCM, AGM, PS, JJL and CEA were involved in study conceptualization. CEA also developed and validated multiplex quantitative IF staining methodology, analyzed IF data and verified the adequacy of tumor in FFPE samples. SHS conceived the study, performed the experimental design, oversaw the project, wrote and edited the final manuscript. JJL, CEA and SHS acquired funding to perform this research. All authors read and approved the manuscript.

\section{Acknowledgements}

We particularly acknowledge all patients for their participation and V. Villar (Biobank, University of Navarra), Dr. D. Alignani and I. Rodriguez (Flow Cytometry Unit, CIMA) and Dr. A. Jimenez-Arranz (Unidad de Genómica, IMIBIC, Cordoba, Spain), for their collaboration in this study. Members of Biobank of University of Navarra and Hospital Costa del Sol, and Genomics Unit of CIMA are also acknowledged. We are grateful to Dr. P.W. Miller for English editing.

\section{References}

[1] Goff SL, Dudley ME, Citrin DE, Somerville RP, Wunderlich JR, Danforth DN, et al. Randomized, Prospective Evaluation Comparing Intensity of Lymphodepletion Before Adoptive Transfer of TumorInfiltrating Lymphocytes for Patients With Metastatic Melanoma. J Clin Oncol 2016;34:2389-97. https://doi.org/10.1200/JC0.2016.66.7220.

[2] Stevanović S, Draper LM, Langhan MM, Campbell TE, Kwong ML, Wunderlich JR, et al. Complete regression of metastatic cervical cancer after treatment with human papillomavirus-targeted tumorinfiltrating T cells. J Clin Oncol 2015;33:1543-50. https://doi.org/10.1200/JC0.2014.58.9093.

[3] Sato E, Olson SH, Ahn J, Bundy B, Nishikawa H, Qian F, et al. Intraepithelial CD8+ tumor-infiltrating lymphocytes and a high $\mathrm{CD} 8+/$ regulatory $\mathrm{T}$ cell ratio are associated with favorable prognosis in ovarian cancer. Proc Natl Acad Sci U S A 2005;102:18538-43. https://doi.org/10.1073/pnas.0509182102.

[4] Westergaard MCW, Andersen R, Chong C, Kjeldsen JW, Pedersen M, Friese C, et al. Tumour-reactive T cell subsets in the microenvironment of ovarian cancer. $\mathrm{Br} J$ Cancer 2019;120:424-34. https://doi.org/10.1038/s41416-019-0384-y.

[5] Aoki Y, Takakuwa K, Kodama S, Tanaka K, Takahashi M, Tokunaga A, et al. Use of adoptive transfer of tumor-infiltrating lymphocytes alone or in combination with cisplatin-containing chemotherapy in patients with epithelial ovarian cancer. Cancer Res 1991;51:1934-9.

[6] Freedman RS, Edwards CL, Kavanagh JJ, Kudelka AP, Katz RL, Carrasco CH, et al. Intraperitoneal adoptive immunotherapy of ovarian carcinoma with tumor-infiltrating lymphocytes and low-dose recombinant interleukin-2: A pilot trial. J Immunother 1994;16:198-210.

https://doi.org/10.1097/00002371-199410000-00004. 
[7] Pedersen M, Westergaard MCW, Milne K, Nielsen M, Borch TH, Poulsen LG, et al. Adoptive cell therapy with tumor-infiltrating lymphocytes in patients with metastatic ovarian cancer: a pilot study. Oncoimmunology 2018;7:e1502905. https://doi.org/10.1080/2162402X.2018.1502905.

[8] Rothermel LD, Sabesan AC, Stephens DJ, Chandran SS, Paria BC, Srivastava AK, et al. Identification of an Immunogenic Subset of Metastatic Uveal Melanoma. Clin Cancer Res 2016;22:2237-49. https://doi.org/10.1158/1078-0432.CCR-15-2294.

[9] Besser MJ, Shapira-Frommer R, Treves AJ, Zippel D, Itzhaki O, Schallmach E, et al. Minimally cultured or selected autologous tumor-infiltrating lymphocytes after a lympho-depleting chemotherapy regimen in metastatic melanoma patients. J Immunother (Hagerstown, Md 1997) 2009;32:415-23. https://doi.org/10.1097/CJI.0b013e31819c8bda [doi].

[10] Dudley ME, Gross CA, Langhan MM, Garcia MR, Sherry RM, Yang JC, et al. CD8+ enriched "Young" tumor infiltrating lymphocytes can mediate regression of metastatic melanoma. Clin Cancer Res 2010;16:6122-31. https://doi.org/10.1158/1078-0432.CCR-10-1297.

[11] Gros A, Robbins PF, Yao X, Li YF, Turcotte S, Tran E, et al. PD-1 identifies the patient-specific CD8(+) tumor-reactive repertoire infiltrating human tumors. J Clin Invest 2014;124:2246-59. https://doi.org/10.1172/JCl73639 [doi].

[12] Ye Q, Song DG, Poussin M, Yamamoto T, Best A, Li C, et al. CD137 accurately identifies and enriches for naturally occurring tumor-reactive T cells in tumor. Clin Cancer Res 2014;20:44-55. https://doi.org/10.1158/1078-0432.CCR-13-0945 [doi].

[13] Fernandez-Poma SM, Salas-Benito D, Lozano T, Casares N, Riezu-Boj J-I, Mancheño U, et al. Expansion of Tumor-Infiltrating CD8 ${ }^{+} \mathrm{T}$ cells Expressing PD-1 Improves the Efficacy of Adoptive T-cell Therapy. Cancer Res 2017;77:3672-84. https://doi.org/10.1158/0008-5472.CAN-17-0236.

[14] Jing W, Gershan JA, Blitzer GC, Palen K, Weber J, McOlash L, et al. Adoptive cell therapy using PD1+ myeloma-reactive T cells eliminates established myeloma in mice. J Immunother Cancer 2017;5:51. https://doi.org/10.1186/s40425-017-0256-z.

[15] Webb JR, Milne K, Nelson BH. PD-1 and CD103 Are Widely Coexpressed on Prognostically Favorable Intraepithelial CD8 T Cells in Human Ovarian Cancer. Cancer Immunol Res 2015;3:926-35. https://doi.org/10.1158/2326-6066.CIR-14-0239.

[16] Matsuzaki J, Gnjatic S, Mhawech-Fauceglia P, Beck A, Miller A, Tsuji T, et al. Tumor-infiltrating NYESO-1-specific CD8+ T cells are negatively regulated by LAG-3 and PD-1 in human ovarian cancer. Proc Natl Acad Sci U S A 2010;107:7875-80. https://doi.org/10.1073/pnas.1003345107.

[17] Strickland KC, Howitt BE, Shukla SA, Rodig S, Ritterhouse LL, Liu JF, et al. Association and prognostic significance of BRCA1/2-mutation status with neoantigen load, number of tumor-infiltrating 
lymphocytes and expression of PD-1/PD-L1 in high grade serous ovarian cancer. Oncotarget 2016;7:13587-98. https://doi.org/10.18632/oncotarget.7277.

[18] Zhou J, Shen X, Huang J, Hodes RJ, Rosenberg SA, Robbins PF. Telomere length of transferred lymphocytes correlates with in vivo persistence and tumor regression in melanoma patients receiving cell transfer therapy. J Immunol (Baltimore, Md 1950) 2005;175:7046-52.

[19] Rosenberg SA, Yang JC, Sherry RM, Kammula US, Hughes MS, Phan GQ, et al. Durable complete responses in heavily pretreated patients with metastatic melanoma using T-cell transfer immunotherapy. Clin Cancer Res 2011;17:4550-7. https://doi.org/10.1158/1078-0432.CCR-11-0116 [doi].

[20] Ayers M, Lunceford J, Nebozhyn M, Murphy E, Loboda A, Kaufman DR, et al. IFN-y-related mRNA profile predicts clinical response to PD-1 blockade. J Clin Invest 2017;127:2930-40. https://doi.org/10.1172/JCl91190.

[21] Kanchi KL, Johnson KJ, Lu C, McLellan MD, Leiserson MDM, Wendl MC, et al. Integrated analysis of germline and somatic variants in ovarian cancer. Nat Commun 2014;5:3156. https://doi.org/10.1038/ncomms4156.

[22] Chae YK, Anker JF, Carneiro BA, Chandra S, Kaplan J, Kalyan A, et al. Genomic landscape of DNA repair genes in cancer. Oncotarget 2016;7:23312-21. https://doi.org/10.18632/oncotarget.8196.

[23] Gros A, Tran E, Parkhurst MR, llyas S, Pasetto A, Groh EM, et al. Recognition of human gastrointestinal cancer neoantigens by circulating PD-1+ lymphocytes. J Clin Invest 2019;129:49925004. https://doi.org/10.1172/JCl127967.

[24] Inozume T, Hanada K, Wang QJ, Ahmadzadeh M, Wunderlich JR, Rosenberg SA, et al. Selection of CD8+PD-1+ lymphocytes in fresh human melanomas enriches for tumor-reactive $T$ cells. J Immunother (Hagerstown, Md 1997) 2010;33:956-64. https://doi.org/10.1097/CJl.0b013e3181fad2b0 [doi].

[25] Wolfl M, Kuball J, Ho WY, Nguyen H, Manley TJ, Bleakley M, et al. Activation-induced expression of CD137 permits detection, isolation, and expansion of the full repertoire of CD8+ T cells responding to antigen without requiring knowledge of epitope specificities. Blood 2007;110:201-10.

[26] Simoni Y, Becht E, Fehlings M, Loh CY, Koo SL, Teng KWW, et al. Bystander CD8+ T cells are abundant and phenotypically distinct in human tumour infiltrates. Nature 2018;557:575-9. https://doi.org/10.1038/s41586-018-0130-2.

[27] Scheper W, Kelderman S, Fanchi LF, Linnemann C, Bendle G, de Rooij MAJ, et al. Low and variable tumor reactivity of the intratumoral TCR repertoire in human cancers. Nat Med 2019;25:89-94. https://doi.org/10.1038/s41591-018-0266-5.

[28] Duhen T, Duhen R, Montler R, Moses J, Moudgil T, De Miranda NF, et al. Co-expression of CD39 and CD103 identifies tumor-reactive CD8 T cells in human solid tumors. Nat Commun 2018;9:2724. 
https://doi.org/10.1038/s41467-018-05072-0.

[29] Xu Z, Dai J, Wang D, Lu H, Dai H, Ye H, et al. Assessment of tumor mutation burden calculation from gene panel sequencing data. Onco Targets Ther 2019;12:3401-9. https://doi.org/10.2147/OTT.S196638.

[30] Morse CB, Elvin JA, Gay LM, Liao JB. Elevated tumor mutational burden and prolonged clinical response to anti-PD-L1 antibody in platinum-resistant recurrent ovarian cancer. Gynecol Oncol Reports 2017;21:78-80. https://doi.org/10.1016/j.gore.2017.06.013.

[31] Wu HX, Wang ZX, Zhao Q, Wang F, Xu RH. Designing gene panels for tumor mutational burden estimation: The need to shift from "correlation" to "accuracy." J Immunother Cancer 2019;7:206. https://doi.org/10.1186/s40425-019-0681-2.

[32] Chalmers ZR, Connelly CF, Fabrizio D, Gay L, Ali SM, Ennis R, et al. Analysis of 100,000 human cancer genomes reveals the landscape of tumor mutational burden. Genome Med 2017;9. https://doi.org/10.1186/s13073-017-0424-2.

[33] Hugo W, Zaretsky JM, Sun L, Song C, Moreno BH, Hu-Lieskovan S, et al. Genomic and Transcriptomic Features of Response to Anti-PD-1 Therapy in Metastatic Melanoma. Cell 2016;165:3544. https://doi.org/10.1016/j.cell.2016.02.065.

[34] Mehnert JM, Panda A, Zhong H, Hirshfield K, Damare S, Lane K, et al. Immune activation and response to pembrolizumab in POLE-mutant endometrial cancer. J Clin Invest 2016;126:2334-40. https://doi.org/10.1172/JCl84940.

[35] Le DT, Durham JN, Smith KN, Wang H, Bartlett BR, Aulakh LK, et al. Mismatch repair deficiency predicts response of solid tumors to PD-1 blockade. Science (80- ) 2017;357:409-13. https://doi.org/10.1126/science.aan6733.

[36] Wang Z, Zhao J, Wang G, Zhang F, Zhang Z, Zhang F, et al. Comutations in DNA damage response pathways serve as potential biomarkers for immune checkpoint blockade. Cancer Res 2018;78:6486-96. https://doi.org/10.1158/0008-5472.CAN-18-1814.

[37] Tian W, Shan B, Zhang Y, Ren Y, Liang S, Zhao J, et al. Association between DNA damage repair gene somatic mutations and immune-related gene expression in ovarian cancer. Cancer Med 2020. https://doi.org/10.1002/cam4.2849.

[38] Chen PL, Roh W, Reuben A, Cooper ZA, Spencer CN, Prieto PA, et al. Analysis of immune signatures in longitudinal tumor samples yields insight into biomarkers of response and mechanisms of resistance to immune checkpoint blockade. Cancer Discov 2016;6:827-37. https://doi.org/10.1158/2159-8290.CD15-1545. 
[39] Leffers N, Fehrmann RSN, Gooden MJM, Schulze URJ, Ten Hoor KA, Hollema H, et al. Identification of genes and pathways associated with cytotoxic $\mathrm{T}$ lymphocyte infiltration of serous ovarian cancer. $\mathrm{Br} \mathrm{J}$ Cancer 2010;103:685-92. https://doi.org/10.1038/sj.bjc.6605820.

[40] Dangaj D, Bruand M, Grimm AJ, Ronet C, Barras D, Duttagupta PA, et al. Cooperation between Constitutive and Inducible Chemokines Enables T Cell Engraftment and Immune Attack in Solid Tumors. Cancer Cell 2019;35:885-900.e10. https://doi.org/10.1016/j.ccell.2019.05.004.

[41] Sangaletti S, Chiodoni C, Tripodo C, Colombo MP. Common extracellular matrix regulation of myeloid cell activity in the bone marrow and tumor microenvironments. Cancer Immunol Immunother 2017;66:1059-67. https://doi.org/10.1007/s00262-017-2014-y.

[42] Chen L, Gibbons DL, Goswami S, Cortez MA, Ahn YH, Byers LA, et al. Metastasis is regulated via microRNA-200/ZEB1 axis control of tumour cell PD-L1 expression and intratumoral immunosuppression. Nat Commun 2014;5:5241. https://doi.org/10.1038/ncomms6241.

[43] Cristescu R, Mogg R, Ayers M, Albright A, Murphy E, Yearley J, et al. Pan-tumor genomic biomarkers for PD-1 checkpoint blockade-based immunotherapy. Science (80- ) 2018;362. https://doi.org/10.1126/science.aar3593.

[44] Ott PA, Bang YJ, Piha-Paul SA, Abdul Razak AR, Bennouna J, Soria JC, et al. T-cell-inflamed geneexpression profile, programmed death ligand 1 expression, and tumor mutational burden predict efficacy in patients treated with pembrolizumab across 20 cancers: KEYNOTE-028. J Clin Oncol 2019;37:318-27. https://doi.org/10.1200/JC0.2018.78.2276.

[45] McGranahan N, Furness AJS, Rosenthal R, Ramskov S, Lyngaa R, Saini SK, et al. Clonal neoantigens elicit $T$ cell immunoreactivity and sensitivity to immune checkpoint blockade. Science 2016;351:1463-9. https://doi.org/10.1126/science.aaf1490.

[46] Bobisse S, Genolet R, Roberti A, Tanyi JL, Racle J, Stevenson BJ, et al. Sensitive and frequent identification of high avidity neo-epitope specific CD8 + T cells in immunotherapy-naive ovarian cancer. Nat Commun 2018;9:1092. https://doi.org/10.1038/s41467-018-03301-0.

[47] Deniger DC, Pasetto A, Robbins PF, Gartner JJ, Prickett TD, Paria BC, et al. T-cell responses to TP53 "Hotspot" Mutations and unique neoantigens expressed by human ovarian cancers. Clin Cancer Res 2018;24:5562-73. https://doi.org/10.1158/1078-0432.CCR-18-0573.

\section{Additional Material}

Additional file 1 (pdf)

Table S1. Patient's clinical and cytological characteristics

Table S2. Number of cells isolated from fresh tumors and fold expansion after REP. 
Figure S1. Study flow Chart.

Figure S2. Image analysis using Inform software.

Figure S3. Multiplex IF Validations.

Figure S4. Gating strategy and example of data from patient P05.

Figure S5. Percentage and phenotypic traits of CD4 and CD8 TILs in the fresh tumor.

Figure S6. Alterations in DNA damage repair (DDR) genes seemed to be associated with the higher TMB and the tumor-reactivity of the final TIL product.

Figure S7. Transcriptome traits of patients with tumor-reactive TIL products.

\section{Supplementary Methods}

Additional file 2 (xls)

Table S3. Variants identified across the patients studied

Table S4. List of non-synonymous somatic mutations identified across the patients studied

Table S5: TMB values across the patients studied.

Table S6. Resume of non-synonymous somatic mutations identified across the patients studied

Table S7. List of genes involved in direct DNA repair pathways that were mutated in the patients studied

Table S8. Resume of DNA repair genes that were mutated in the patients studied

Table S9. Distribution of variants in genes of the direct DNA repair machinery across patients and different pathways.

Table S10. DNA repair gene mutations across the patients studied.

Appendix 1. DNA repair genes in cancer

Appendix 2. Genes included in the TruSight Tumor 170 panel

\section{Figures}



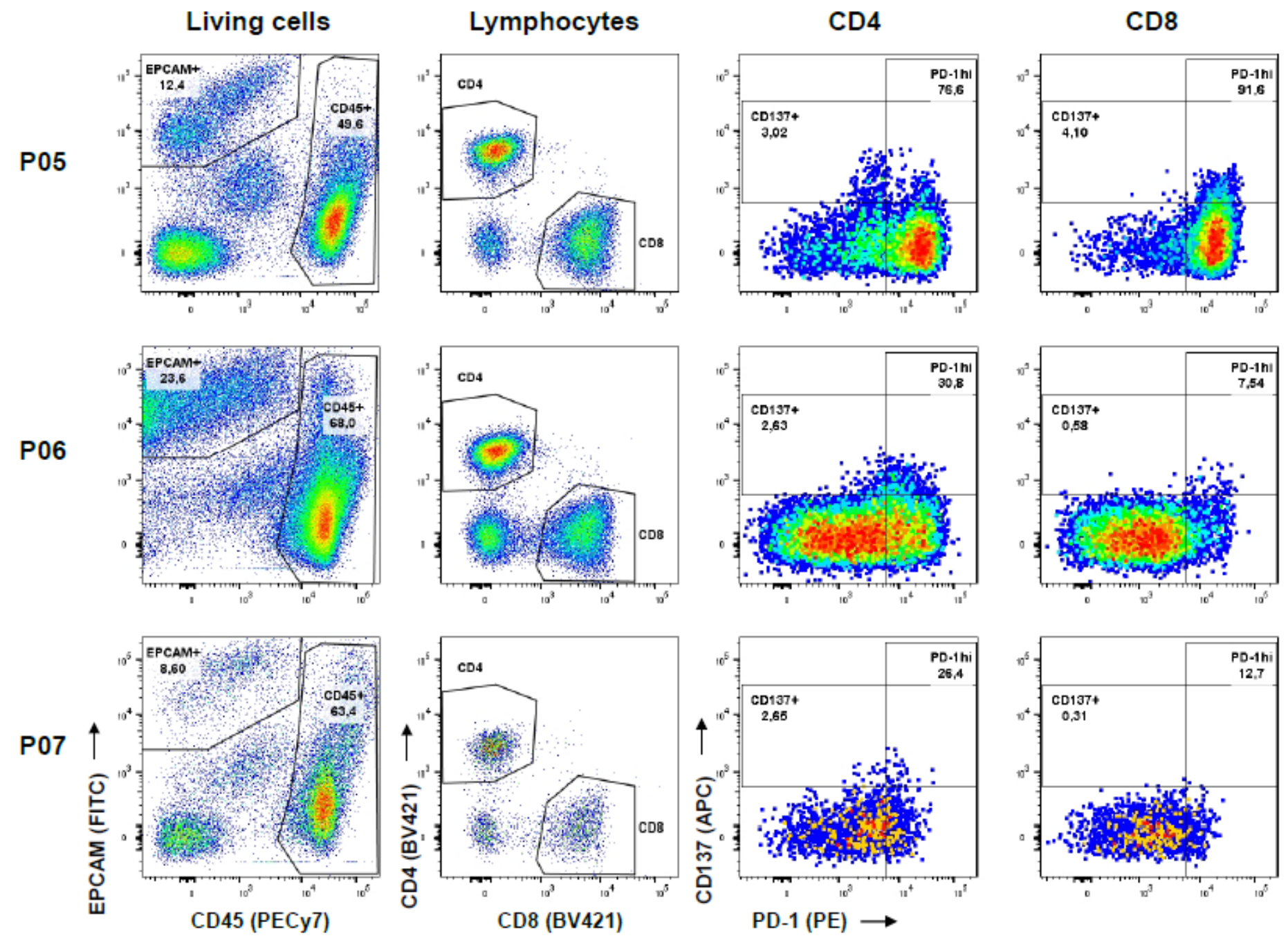

Figure 1

PD-1 and CD137 expression in CD4+ and CD8+ TILs in tumor samples from OC patients. Tumor singlecell suspensions were analyzed by FC as detailed in Material and methods. Gating strategy is described in Additional file 1: Fig. S4. Figure shows three representative patients (P05, P06 and P07). Names at the top indicate the parental population. Numbers indicate the percentage of gated cells with respect to the parental population. 
A
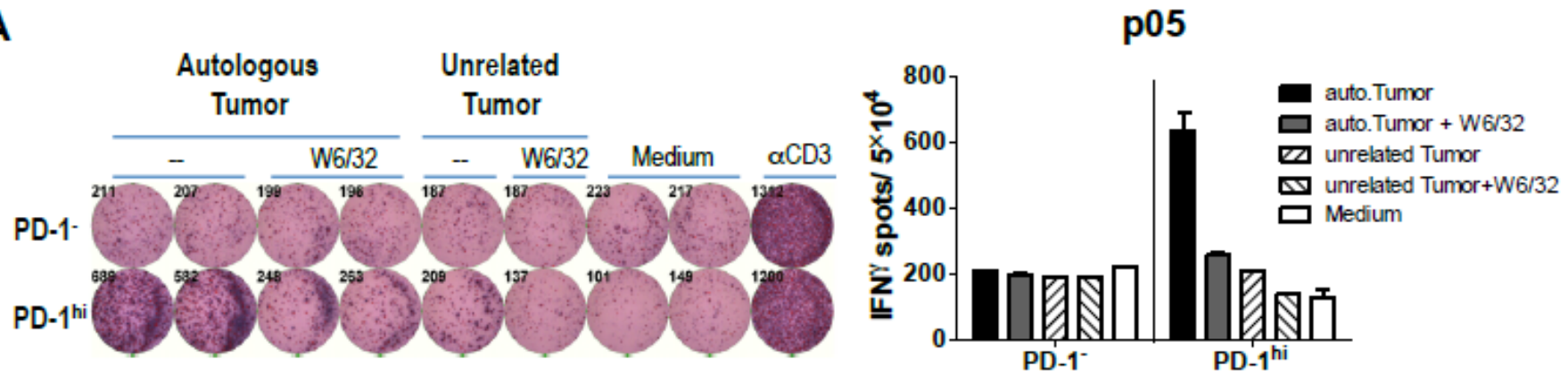

B
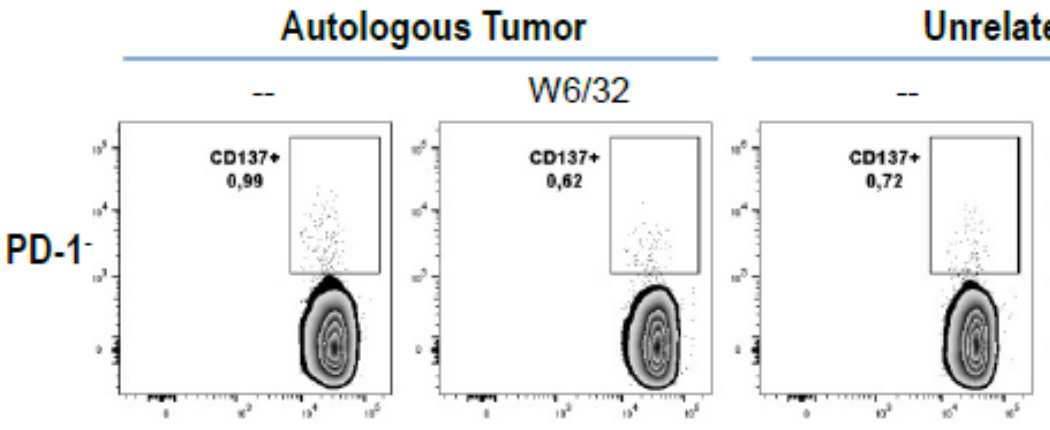

Unrelated Tumor
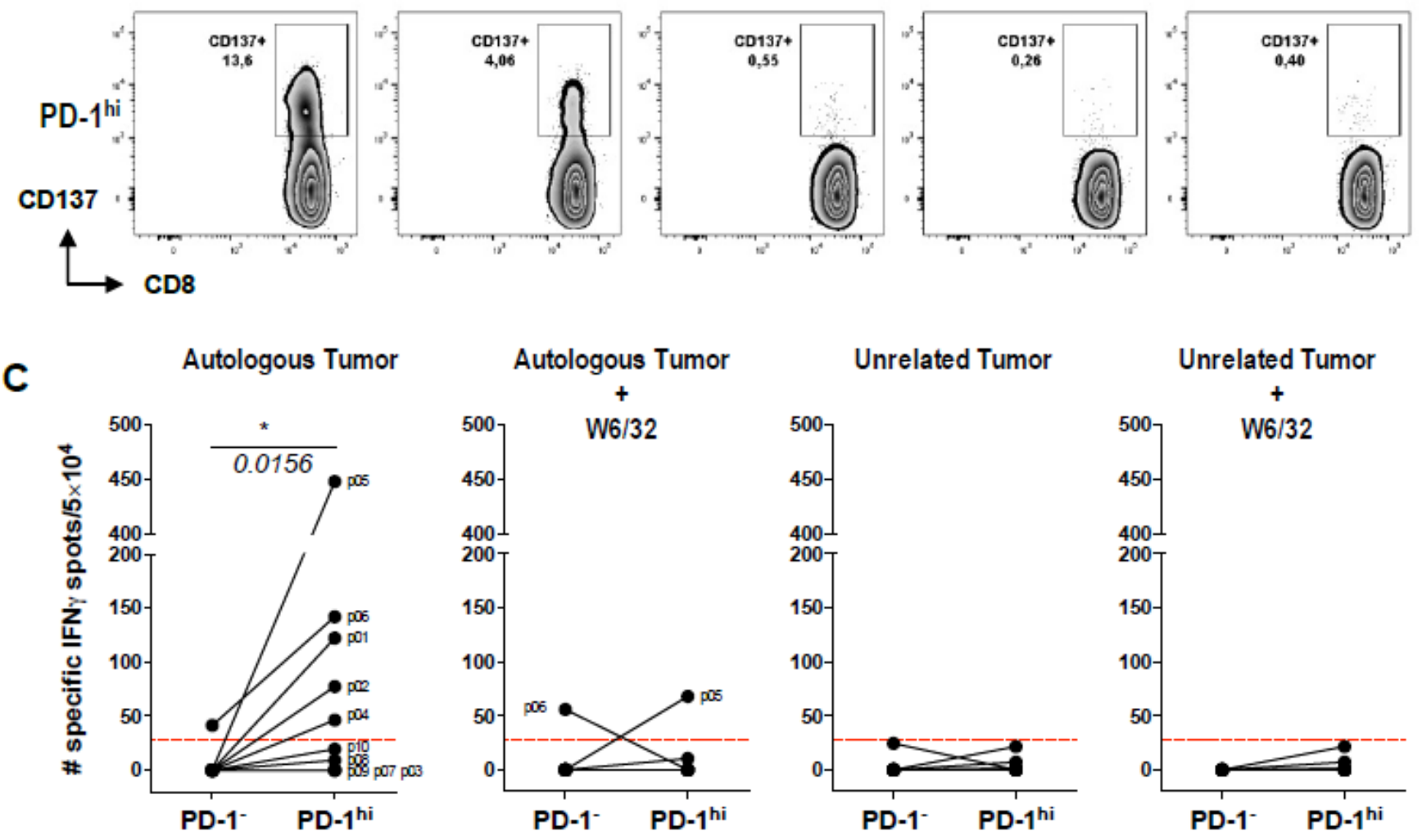

Figure 2

Cells derived from the PD-1hi CD8 TIL subset recognize autologous tumor cells. Cells expanded from the PD-1- or the PD-1hi CD8 TIL subset isolated from different patients were co-cultured with the respective autologous tumor cells, or with unrelated tumor cells (H929), in the presence or absence of HLA-I blocking antibody (W6/32) and tumor recognition was assessed by measuring IFN- $\gamma$ release by ELISPOT ( $A$ and $C$ ) and the frequency of CD137+ cells by FC (B). (A and B) Antitumor reactivity of cells derived from PD-1- 
and PD-1hi CD8+ TILs from patient P05. (A) Left: picture of the ELISPOT plate. Plate-bound anti-CD3 mAb was used as a positive control. Right: the graph shows the number of spots per $5 \otimes 104$ cells (mean $\pm S D$ ).

(B) Dot plots display the frequency of CD137+ cells in PD-1- (up) and PD-1hi (down) derived CD8 TILs 24 hours after co-culture with target cells. Cells are gated on blastic CD45+CD3+CD8+cells. (C) Antitumor reactivity of cells derived from PD-1- and PD-1hi CD8+ TILs from each patient. The number of targetspecific IFN- $\gamma$ spots was determined by calculating the difference between the number of spots generated in the presence of target cells (autologous tumor or nonrelated tumor) and in their absence (TIL alone). Reactivities $>30$ specific IFN- $y$ spots and twice the background were considered positive (horizontal dotted line). Each line matches the PD-1- and PD-1hi TIL products derived from the same patient. Mean $\pm S D$. ${ }^{*} \leq \leq 0.05$. Wilcoxon matched-pairs signed rank test, two-tailed, $95 \%$ confidence level.
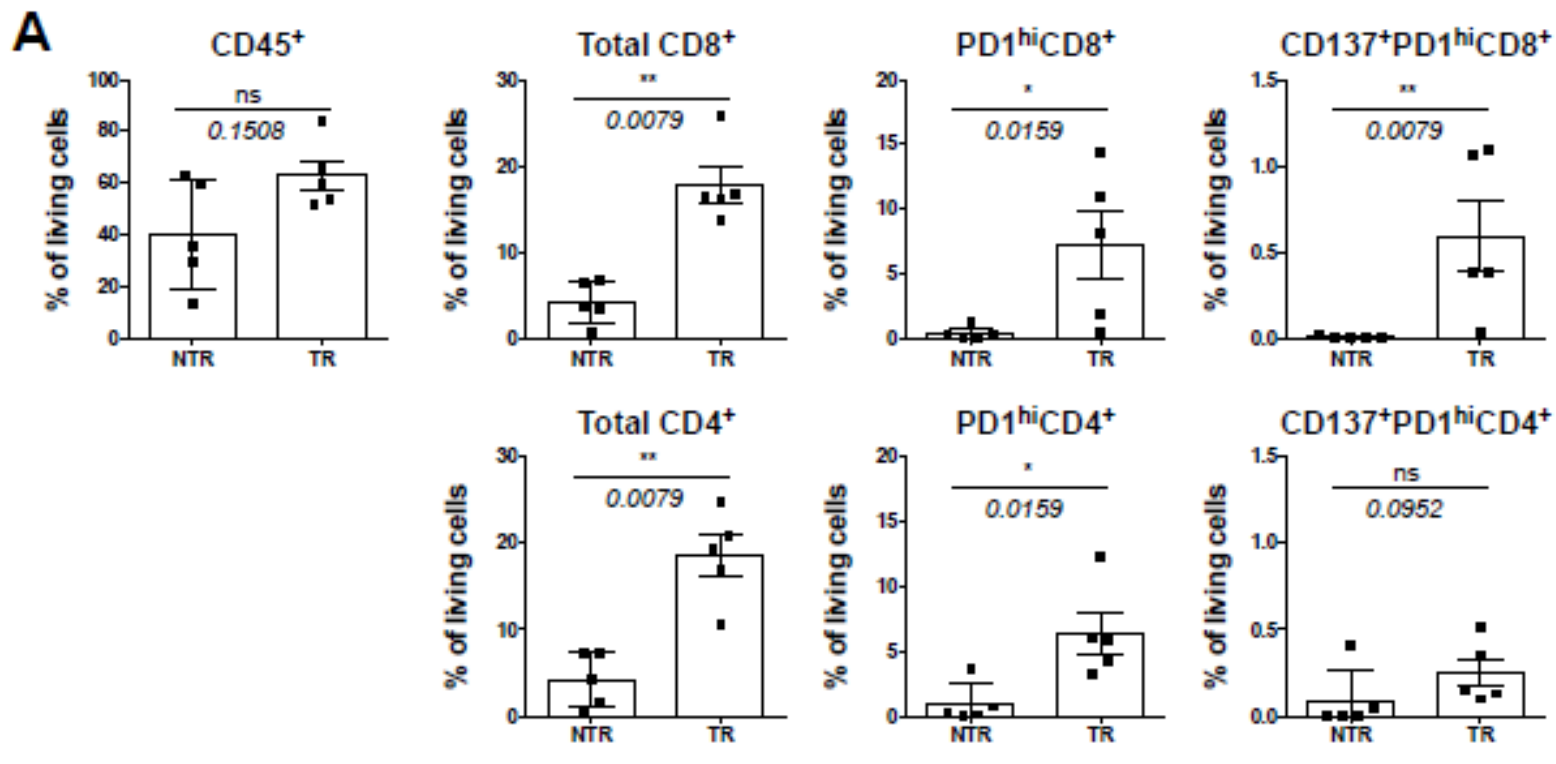

\section{B}
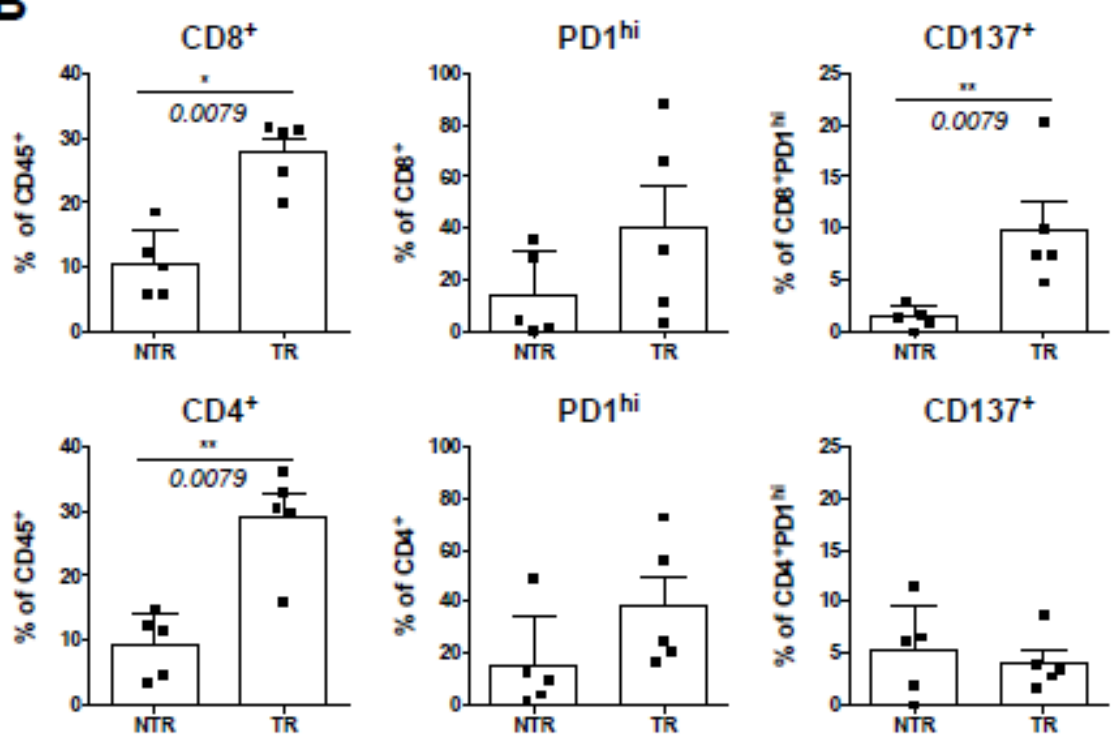

Figure 3 
Detection of CD137+ cells within the PD-1hiCD8+ TIL subset in the fresh tumor correlates with antitumor reactivity of the final TIL product. The phenotypic traits of CD4 and CD8 TILs in the fresh tumor (Additional file 1: Fig. S5) from patients who rendered TR TILs were compared with those from patients with NTR TIL products. (A) Frequency of the indicated subsets in living cells (Zombi NIR-). (B) Frequency of $\mathrm{CD} 8+, \mathrm{CD} 4+, \mathrm{PD}-1 \mathrm{hi}$ and $\mathrm{CD} 137+$ cells in the respective parental population. Each dot represents 1 patient's sample. Mean $\pm S E M$. $* P \leq 0.05 ; * * P \leq 0.01$, Mann-Whitney Test, two-tailed, $95 \%$ confidence level.

A
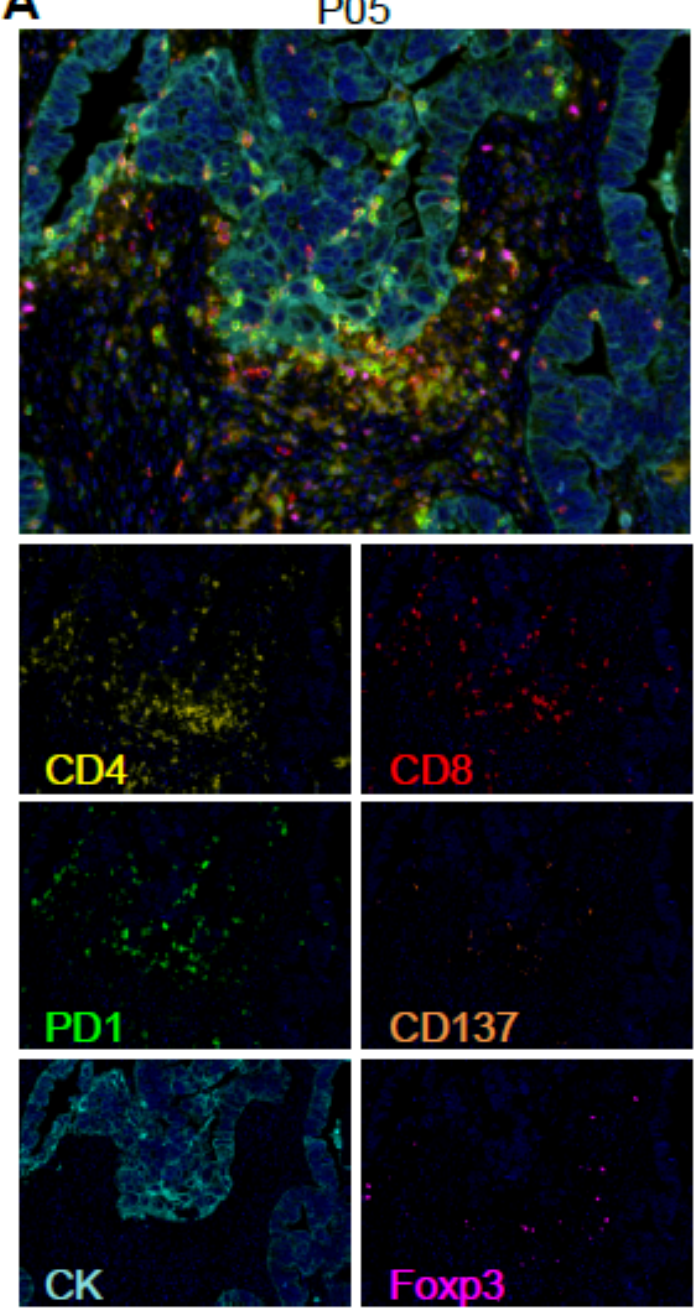
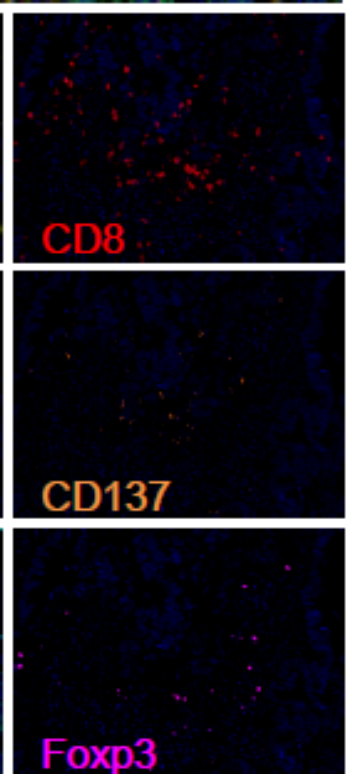

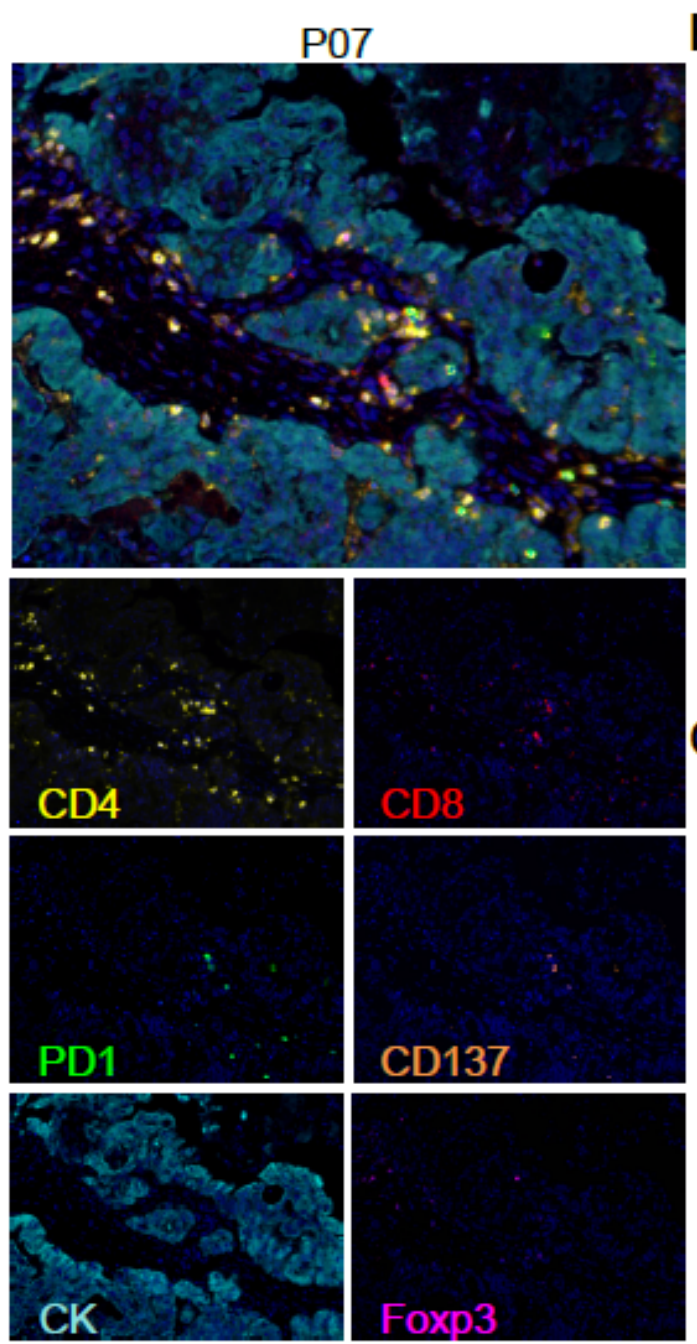

B
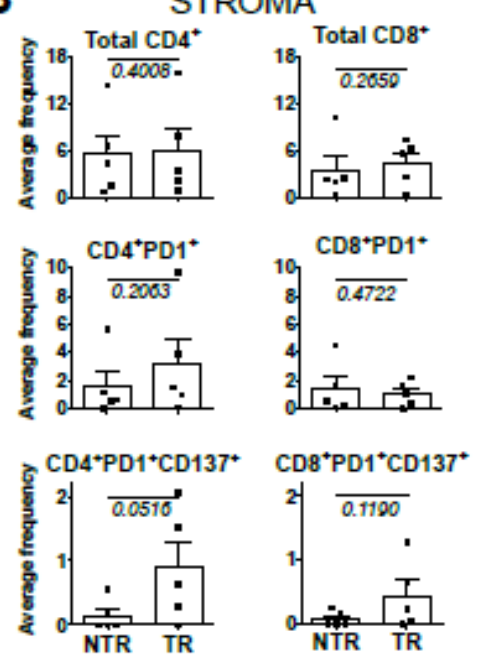

C
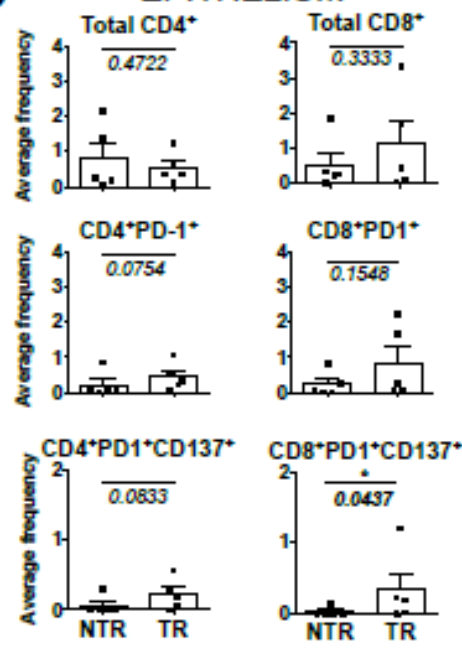

\section{Figure 4}

Multiplex immunofluorescence assessment of different TIL subsets in the tumor microenvironment. Tumors were analyzed by multiplexed IF using mAbs targeting CK (Dark Blue), CD4 (yellow), CD8 (red), PD-1 (green), CD137 (orange) and Foxp3 (magenta) molecules. (A) Representative fluorescence images corresponding to patient P05 (TR group) and patient 07 (NTR group). Immunofluorescent multiplexing images were scanned with a spectral scanner (Polaris) using 20x magnification. Figures at the top compile the entire staining (merge). ( $B$ and $C$ ) Average frequency with respect to total cells of different TIL subsets in the stroma (B) and in the epithelium (C) of tumors. Total CD8+ and CD4+ cells, PD-1+CD8+ 
and PD-1+CD4+ cells (regardless of whether or not they express CD137) and CD137+PD-1+CD 8+ and CD137+PD-1+CD4+ cells were quantified using multiplexed quantitative analysis. Patients who rendered TR TILs were compared with those that did not render TR TILs (NTR). No significant differences were found in the percentage of Foxp3+ TIL, neither in the epithelium or in the stroma, between both groups (data not shown). Each dot represents 1 patient. Mean \pm SEM. Mann-Whitney Test, one-tail, $90 \%$ confidence level, Monte Carlo exact p-value estimation.

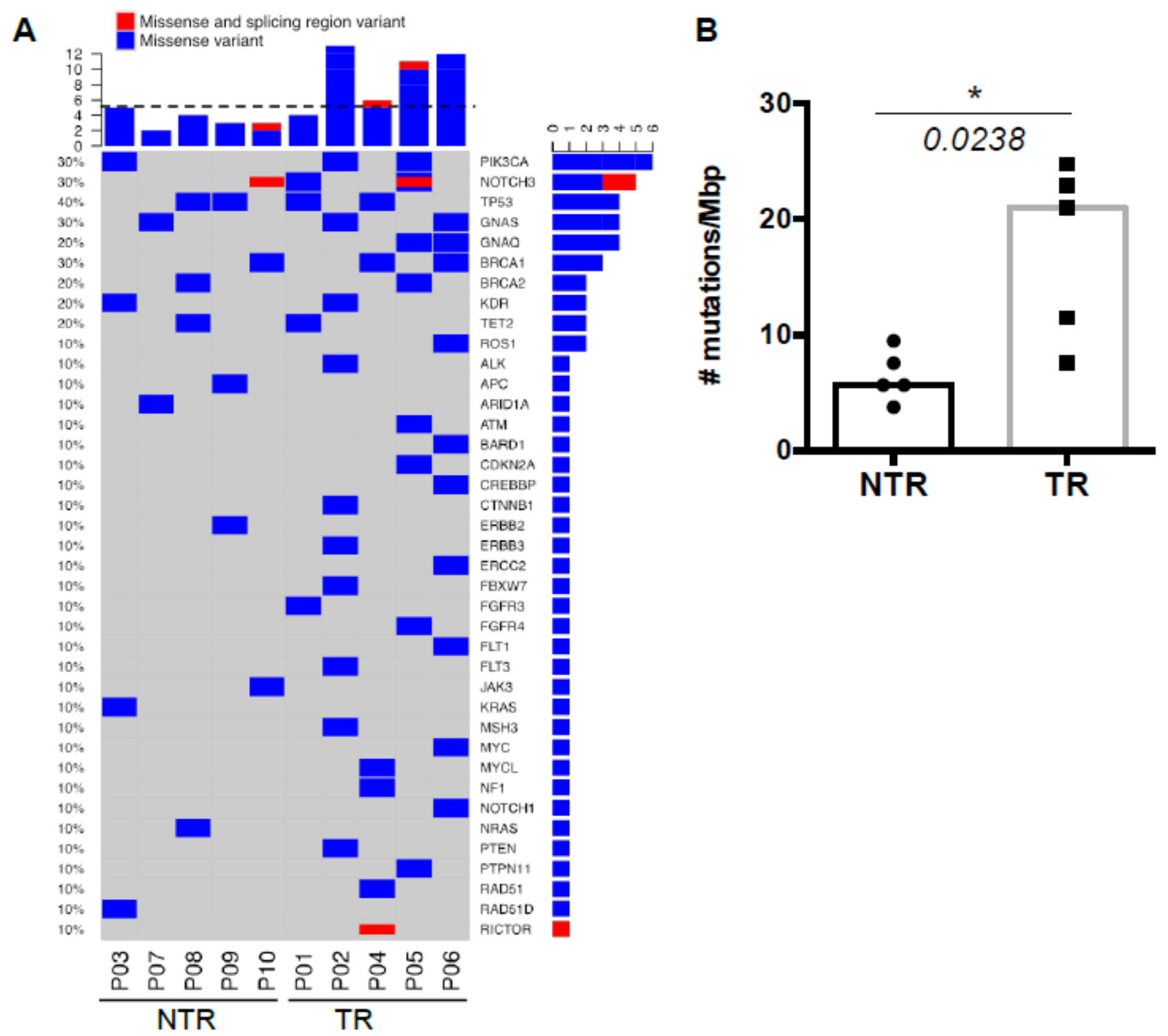

Figure 5

Patients rendering TR TILs exhibited higher predicted TMB than those with NTR TILs. (A) Oncoprint displaying nonsynonymous somatic mutations in 39 cancer genes across studied patients. These genes are those of the TST170 panel in which mutations were found. $95.2 \%$ of these mutations were missense 
variants (60/63) (Additional file 2: Table S6). The digits on the left indicate the percentage of patients who presented mutation in the indicated gene. The bars on the right show the total the number of mutations in each gene. (B) Estimation of TMB with TST170 panel. Patients who rendered TR TILs were compared with those with NTR TILs. Each dot represents 1 patient. Mann-Whitney Test, two-tailed, 95\% confidence level.

A

TR vs NTR patients

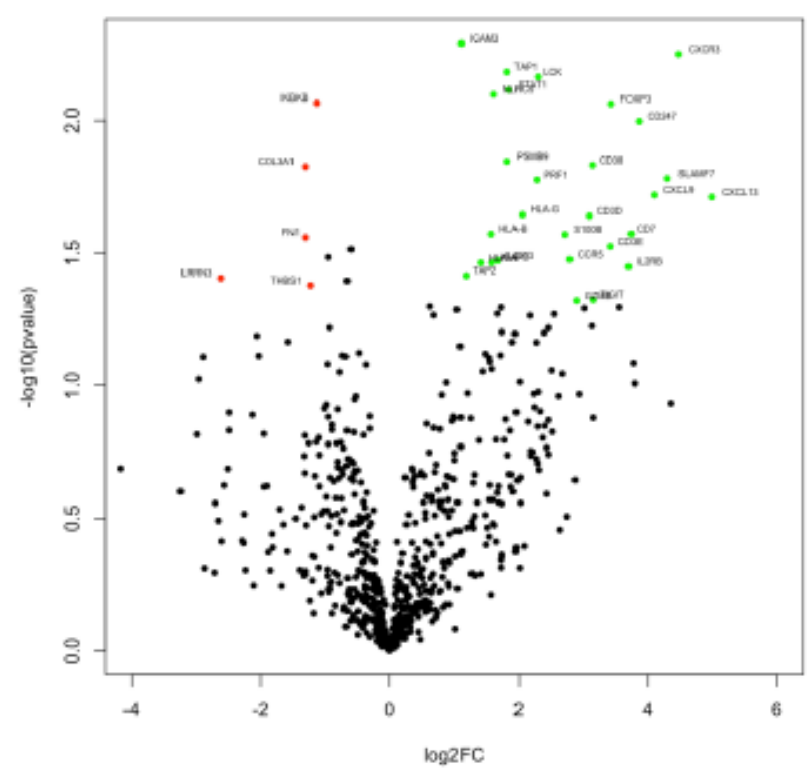

B

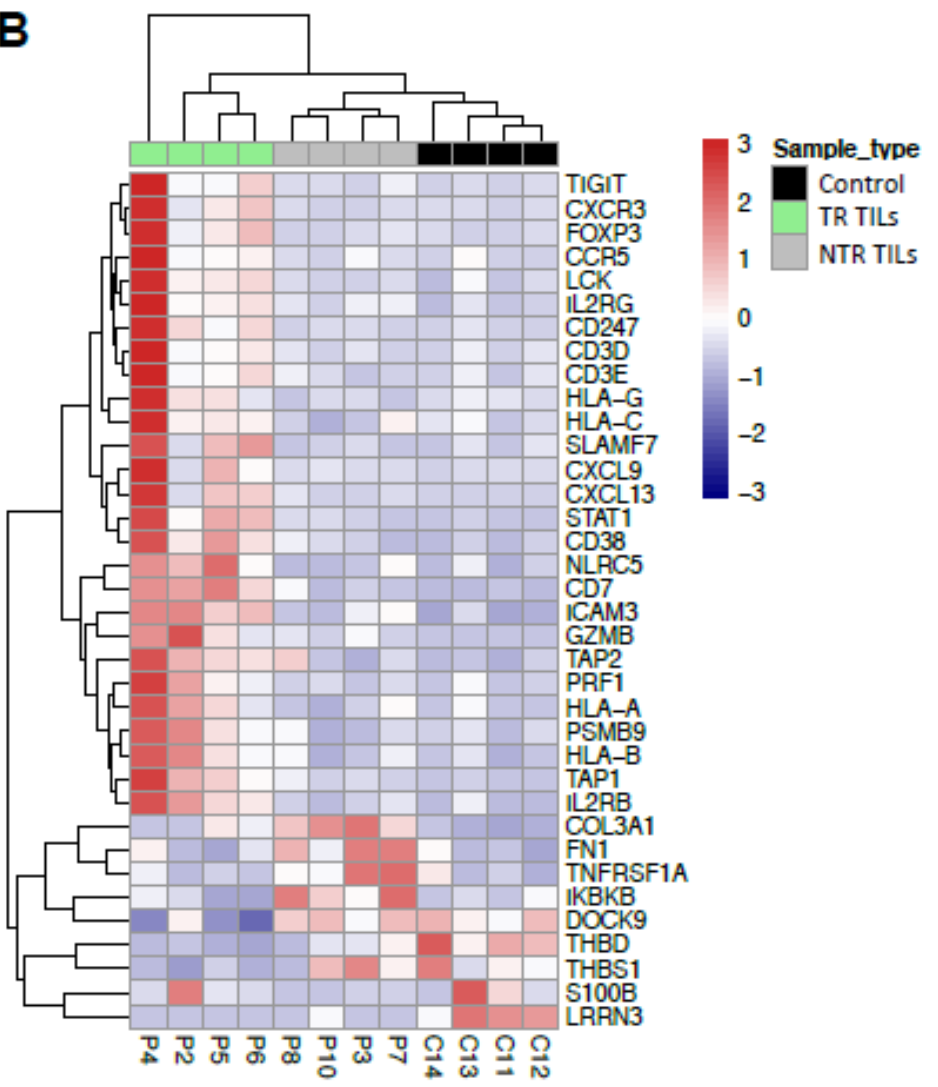

C

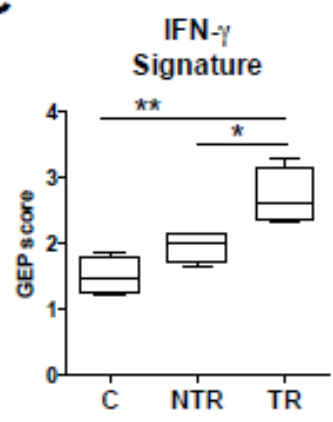

Expanded immune

Signature

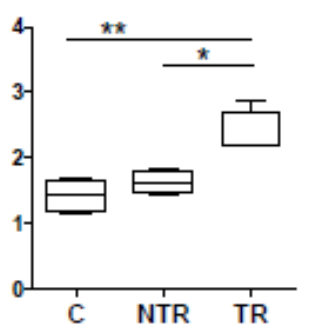

T cell Inflamed Signature

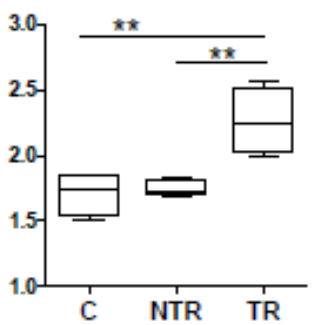

D

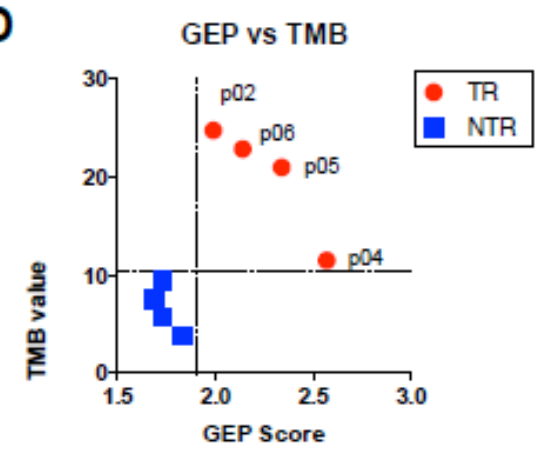

\section{Figure 6}

Immune activation gene signature in baseline tumor samples distinguishes patients rendering TR TIL. The expression of 770 immune-related genes was profiled using the PanCancerlmmune Profiling Panel. Only FFPE-tumor RNA samples that met the quality control requirements Ipercentage of 300 nucleotide-

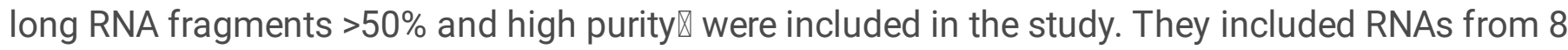
patients (P02, P04, P05 and P06, from TR group, and P03, P07, P08 and P10, from NTR group) and 4 
ovaries with non-malignant disease (C11-C14). (A) Volcano plot for DEG between the TR and NTR groups. The colored circles indicate significant DEG $(p<0.05)$ with fold change $>2$ (green) or $<0.5$ (red). (B) Heatmap showing the assortment of patients and healthy ovaries (based on the top 36 DEG between TR and NTR groups). Rows represent genes and columns patients and controls. Gene expression was standardized by the mean of the samples. The rows and columns have been grouped using unsupervised hierarchical clustering. (C) Gene expression signatures associated with TR TIL products. GEP score was calculated by averaging the expression level of the genes included in the IFN- $\gamma$ signature, Expanded immune signature and T-cell inflamed signature. (D) GEP score was plotted against the TMB value in

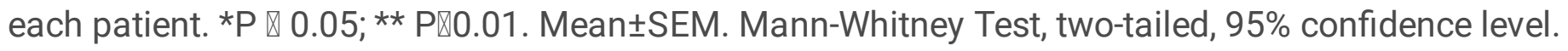

\section{Supplementary Files}

This is a list of supplementary files associated with this preprint. Click to download.

- 04AdditionalFile2TableS3toS10Appendix12JECCR.xlsx

- 03AdditionalFile1JECCR2.pdf

- 03AdditionalFile1JECCR2.pdf

- 04AdditionalFile2TableS3toS10Appendix12JECCR.xIsx 